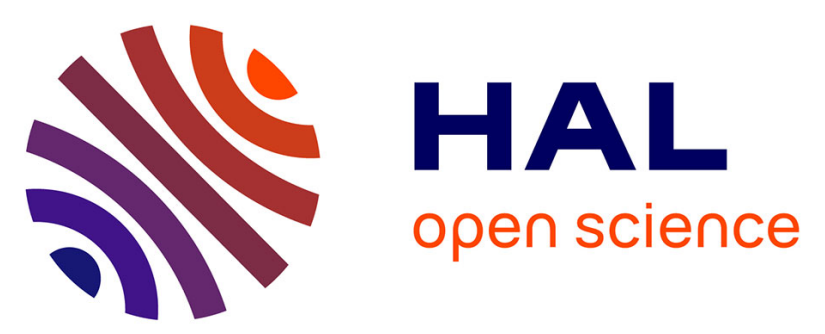

\title{
Les juges constitutionnels face au Traité de Lisbonne, contribution à l'étude comparée des décisions des Cours constitutionnelles française, allemande et tchèque Safia Cazet
}

\section{- To cite this version:}

Safia Cazet. Les juges constitutionnels face au Traité de Lisbonne, contribution à l'étude comparée des décisions des Cours constitutionnelles française, allemande et tchèque. Annuaire International de Justice Constitutionnelle, 2010, XXVI, pp.43-79. hal-02292142

HAL Id: hal-02292142

https://hal.univ-reunion.fr/hal-02292142

Submitted on 19 Sep 2019

HAL is a multi-disciplinary open access archive for the deposit and dissemination of scientific research documents, whether they are published or not. The documents may come from teaching and research institutions in France or abroad, or from public or private research centers.
L'archive ouverte pluridisciplinaire HAL, est destinée au dépôt et à la diffusion de documents scientifiques de niveau recherche, publiés ou non, émanant des établissements d'enseignement et de recherche français ou étrangers, des laboratoires publics ou privés. 


\title{
LES JUGES CONSTITUTIONNELS FACE AU TRAITE DE LISBONNE
}

\author{
Contribution à l'étude comparée des décisions \\ des Cours constitutionnelles française, allemande et tchèque
}

\author{
par Safia CAZET *
}

Le traité de Lisbonne est entré en vigueur le 1er décembre 2009 soit deux ans après sa signature le 13 décembre 2007. Le processus de ratification a été houleux, rythmé par un référendum négatif ${ }^{1}$, par un recours majoritaire à la voie parlementaire, dont l'un qualifié de "coup d'État ${ }^{2}$ et par la saisine de certaines Cours constitutionnelles dont les décisions sont loin d'être neutres.

La saisine de la juridiction constitutionnelle n'est pas automatique. En réalité, peu de juridictions constitutionnelles ont été saisies: le

* Docteur en droit public de l'Université de la Réunion, membre du Centre de Recherche juridique.

L'auteur adresse ses plus vifs remerciements aux Professeurs Patrick Gaïa et Denys Simon pour leurs conseils et leur indéfectible soutien.

1 Le peuple irlandais a voté "non" le 12 juin 2008 à 53,4 \% avec 53\% de participation.

2 A.-M. Le Pourhiet, Ce qui fonde la qualification de coup d'État, Les Cahiers de I'Indépendance, $\mathrm{n}^{\circ}$ 4, 2007, p. 44, N. Dupont-AignAN, Le coup d'État simplifié, Paris, Ed. du Rocher, 2008. 
Conseil constitutionnel français ${ }^{3}$, la Cour constitutionnelle fédérale allemande ${ }^{4}$, la Cour constitutionnelle tchèque ${ }^{5}$, la Cour constitutionnelle de la République de Lettonie ${ }^{6}$, la Cour constitutionnelle autrichienne ${ }^{7}$ et la Cour constitutionnelle belge ${ }^{8}$. L'étude porte sur les décisions française, allemande et tchèque.

Les décisions divergent et convergent. Elles convergent sur un point. Elles n'ont pas constitué un obstacle dirimant à la ratification du traité de Lisbonne. La décision française d'inconstitutionnalité a été surmontée par une loi de révision constitutionnelle adoptée par le Congrès le 4 février $2008^{9}$. En Allemagne, bien que le traité de Lisbonne ait été déclaré conforme à la Loi fondamentale, la ratification n'a été possible qu'après l'adoption des nouvelles lois d'accompagnement ${ }^{10}$ conformes aux prescriptions de la décision du 30 juin 2009. En République tchèque, la Cour constitutionnelle a déclaré le traité de Lisbonne compatible avec l'ordre constitutionnel. Mais le Président de la République Vaclav Klaus, particulièrement euro-sceptique, et les sénateurs, visiblement mécontents de la première décision, ont déposé une seconde requête constitutionnelle, ce qui a retardé le dépôt de l'instrument de ratification au 13 novembre 2009.

Pour le reste, les décisions divergent tant sur la forme que sur le fond. Avant de passer à l'étude des différences sur le fond, il est nécessaire de s'attarder sur les dissemblances formelles qui tiennent à la nature du contrôle, au type de recours et à l'étendue des contrôles. En effet, la façon dont les juges constitutionnels abordent le contrôle de constitutionnalité influence pour partie les solutions retenues au fond.

Les juges constitutionnels tchèque, allemand et français ont opéré un contrôle a priori de compatibilité entre leur Constitution et le traité de Lisbonne.

En France, comme en République tchèque, est organisé un contrôle a priori de compatibilité entre le traité et la Constitution. Cette décision de compatibilité conditionne dans les deux cas la ratification. En

3 CC, 560 DC, 20 décembre 2007, Traité modifiant le traité sur l'Union européenne et le traité instituant la Communauté européenne.

4 BVerfG, 30 juin 2009, II. Senat, 2 BvE 2/08, 2 BvE 5/08, 2 BvR 1010/08, 2 BvR 1022/08, 2 BvR 1259/08, 2 BvR 182/09, Absatz-Nr.1-421

Décision du 26 novembre 2008, aff. PI. ÚS n 19/08.

Cour constitutionnelle de Lettonie, Riga, 7 avril 2009, aff. $n^{\circ}$ 2008-35-01.

Cour constitutionnelle autrichienne, 30 septembre 2008, SV 2 / 08 et G 80/08.

Trois recours ont été introduits : Cour constitutionnelle belge, 19 mars 2009, $n^{\circ} 58 / 2009$; 16 juillet $2009, n^{\circ} 125 / 2009 ; 13$ octobre $2009, n^{\circ}$ 156/2009.

9 Loi n² 2008-103, 4 févr. 2008, Journal Officiel 5 Février 2008.

10 Elles ont été adoptées par le Bundestag le 8 septembre 2009 et ratifiées par le Bundesrat le 18 septembre 2009. 
France, en effet, le Conseil constitutionnel a été saisi par le Président de la République, sur le fondement de l'article 54 de la Constitution, de la question de savoir si l'autorisation de ratifier le traité de Lisbonne doit être précédée d'une révision de la Constitution. En République tchèque, le Sénat a déféré le traité sur le fondement de l'article $87 \S 2$ de la Constitution dans le même but. En Allemagne, la Cour a été saisie de la "loi d'accord " qui reprend l'ensemble du texte du traité et des "lois d'accompagnement " qui organisent les droits de participation et de contrôle accordés aux parlements nationaux dans le cadre du nouveau traité. La ratification du traité était suspendue à une déclaration de compatibilité avec la Loi fondamentale.

Si ce sont tous des contrôles a priori de constitutionnalité, la nature des recours est pourtant différente. En effet, en Allemagne, deux types de recours ont été déposés: des plaintes constitutionnelles par des requérants individuels et des recours inter-organiques (Organstreit) déposés par des membres du Bundestag au nom du Bundestag. Ces recours supposent la violation d'un droit subjectif. A été invoquée la violation du droit à l'autodétermination démocratique fondé sur l'article 38.1 de la Loi fondamentale ${ }^{11}$. La nature du recours est donc subjective. Un recours objectif est pourtant possible en droit allemand. II est réservé au gouvernement fédéral, à tout gouvernement d'un Länder, et à un tiers des membres du Bundestag. Tel n'est pas le recours introduit en l'espèce. Et pourtant, la Cour a saisi cette occasion pour opérer un contrôle abstrait du traité ${ }^{12}$. En droits français et tchèque, les recours sont objectifs. Le contrôle vise à déterminer la constitutionnalité d'une norme internationale.

Enfin, l'étendue du contrôle de compatibilité varie. En effet, le juge tchèque a choisi de limiter son contrôle aux dispositions critiquées dans la requête introductive d'instance ${ }^{13}$, qu'il s'agisse de dispositions nouvelles ${ }^{14}$ ou pas. Le requérant a la charge de la preuve et le juge est tenu par la demande ${ }^{15}$. Le juge français a une toute autre attitude. Le

11 X. MAGNON, «Le juge constitutionnel national, dernier obstacle au processus d'intégration européenne ? Interrogation(s) autour d'une lecture de l'arrêt de la Cour constitutionnelle fédérale allemande du 30 juin 2009 sur "le traité de Lisbonne" ", RFDC, 2010, $\mathrm{n}^{\circ} 82$, p. 422.

12 C. TOMUSCHAT, The ruling of the German Constitutional Court on the Treaty of Lisbon, The German Law Journal, vol. 10, $\mathrm{n}^{\circ} 8$, p. 1259.

13 Suivant l'ordre de la requête du Sénat : 2 § 1, 4 § 2, 352 § 1 TFUE, 48 § 6 et 7 TUE, 216 TFUE, 6 § 1 et $\S 2$ TUE, 2 TUE.

14 Le Sénat ne contestait que des dispositions nouvelles: Décision du 26 novembre 2008, aff. PI. ÚS n 19/08, pt 79 à 87.

15 Décision du 26 novembre 2008, aff. PI. ÚS n 19/08, pt 72 à 77, et la décision du 3 novembre 2009, aff. PI ÚS n²9/09, pt. 105 à 114. 
contrôle ne s'étend pas à l'intégralité du traité, il est borné aux seules dispositions nouvelles ${ }^{16}$. Le juge allemand a procédé à un contrôle de l'intégralité du traité ${ }^{17}$, mais il est limité à l'examen de la violation des droits subjectifs des requérants.

En République tchèque, le problème de l'autorité de chose jugée s'est posé lorsqu'un second recours constitutionnel a déposé à l'encontre du traité de Lisbonne. La Cour a décidé de retenir une conception restrictive de la res judicata ${ }^{18}$. Consciente que la res judicata n'empêchera que peu la multiplication de recours proches bien que différents ${ }^{19}$, elle refuse donc de réévaluer les dispositions contestées à l'occasion du premier recours 20.

En France, la portée de la décision constitutionnelle relative au Traité établissant une Constitution pour l'Europe a mis en avant le problème de la chose jugée. Mais ce traité n'étant jamais entré en vigueur, il n'y avait pas vraiment d'obstacle en terme de res judicata. En revanche pour les dispositions identiques, le juge français a utilisé une motivation par référence.

Les juges n'abordent donc pas le traité de Lisbonne sous un angle identique. Mais des éléments de convergence existent. D'une part, l'objet d'étude est le même, le traité de Lisbonne. D'autre part, les Constitutions bien que différentes, protègent des valeurs identiques. Enfin, les requêtes tchèque et allemande soulevaient des moyens d'inconstitutionnalité proches. Ainsi les juges constitutionnels ont dû répondre à la question de savoir si le traité de Lisbonne porte atteinte à la souveraineté des États et au principe démocratique.

\section{I - L'APPRECIATION VARIABLE DE L'ATTEINTE A LA SOUVERAINETE ETATIQUE}

À tous les juges constitutionnels, la question a été posée de déterminer dans quelle mesure le traité de Lisbonne pouvait altérer la souveraineté étatique.

16 CC, 560 DC, 20 décembre 2007, Traité modifiant le traité sur l'Union européenne et le traité instituant la Communauté européenne, pt. 10

17 X. MAGNON, précit., p. 424.

18 Décision du 3 novembre 2009, aff. PI ÚS n²29/09, pt. 96.

19 Ibidem, pt. 98 et 99.

20 Ibidem, pt. 101 à 104. 
La souveraineté a plusieurs sens interdépendants, lesquels ne se suffisent pas à eux-mêmes ${ }^{21}$. Tout d'abord, elle est le plus haut pouvoir que l'on rencontre sur un territoire, en ce sens elle nie toute subordination à un pouvoir externe (indépendance) et à tout pouvoir interne (capacité d'autodétermination). Elle est le caractère suprême d'une puissance étatique ${ }^{22}$. Ensuite, elle est un ensemble de pouvoirs compris dans la puissance. Cette conception matérielle de la souveraineté postule l'existence de compétences dites de souveraineté qui doivent rester dans le giron étatique sous peine pour l'État de perdre sa souveraineté. Ce second sens est lié au premier car si le champ des compétences étatiques est vidé de sa substance, cela entraine de facto la perte de la capacité d'autodétermination ${ }^{23}$. Enfin, au sens organique, la souveraineté renvoie aux personnes qui forment l'organe suprême de puissance d'État. Ainsi, lorsqu'on évoque la souveraineté populaire, on fait référence au corps des citoyens qui possède cette puissance suprême.

Ainsi, pour apprécier si le traité de Lisbonne porte atteinte à la souveraineté des États, deux questions peuvent être posées: dans quelle mesure le traité affecte-t-il la capacité d'autodétermination des États et dans quelle mesure l'importance des transferts vide-t-elle de substance le pouvoir de décider? Mais ces questions n'étaient pas toutes posées aux juges constitutionnels en raison des spécificités des procédures contentieuses 24 .

L'impact des transferts sur la souveraineté étatique constitue la question centrale des trois décisions étudiées.

\section{A - L'évaluation de l'affectation de la capacité d'autodétermination}

21 K. BeCHET-Golovko, "Qu'en est-il du concept de « souveraineté » ? A l'occasion de la décision du Conseil constitutionnel sur le traité de Lisbonne », Politeia 2008, n 13, p. 75 et s.

22 R. CARRe de Malberg, Contribution à la théorie générale de l'État, Tome premier, Paris, Sirey 1920, éd. du CNRS réimprimé en 1962, p. 75.

23 C'est pourquoi il est difficile d'évaluer la perte de souveraineté de façon fractionnée, en examinant successivement l'atteinte portée à chacun des trois sens. Mais la nécessité d'organiser le raisonnement et l'exposé rend inévitable un tel fractionnement.

24 Seule la Cour constitutionnelle fédérale allemande et la Cour constitutionnelle tchèque ont dû répondre à cette question. Cf. BverfG 30 juin 2009, 2 BvE 2/08, Lisbon, § 111 à 113 et décision du 26 novembre 2008, aff. PI. ÚS n¹9/08, pt 19. La question n'a pas été soulevée en tant que telle devant le Conseil constitutionnel, mais il y a implicitement répondu en listant les atteintes aux conditions essentielles d'exercice de la souveraineté. 
La souveraineté correspond à la capacité pour l'État de décider luimême pour son territoire. Cela suppose qu'il ne soit assujetti à aucune puissance étrangère, à aucun pouvoir interne.

Ainsi, pour répondre aux requérants qui suspectaient le traité de Lisbonne d'opérer un transfert de souveraineté au profit de l'Union, le juge allemand s'est attaché à prouver méticuleusement que l'Allemagne conserve cette capacité d'autodétermination. Elle est d'ailleurs la seule à effectuer cet examen de cette façon. Elle explique comment la "substance de l'autorité étatique " est protégée ${ }^{25}$. Son raisonnement repose sur quatre fondements.

En ce qui concerne le premier fondement, la Cour examine les règles de répartition et délimitation des compétences. Sous l'empire du traité de Lisbonne, le principe d'attribution reste la règle ${ }^{26}$. L'Union n'a que des compétences déléguées. L'État membre conserve donc la compétence de la compétence. La catégorisation et la classification des compétences ${ }^{27}$ confortent l'idée que l'Union ne possède que des compétences limitées qui ne peuvent pas être étendues de façon autonome. Bien évidemment, la catégorisation n'est pas d'une clarté totale et il existe des compétences qui se trouvent hors de cette classification, comme l'existence de compétences parallèles, la PESC, la coordination des politiques économiques et de l'emploi, la méthode ouverte de coordination. La Cour estime que ces éléments qui permettent une extension des compétences n'affectent pas le principe d'attribution limitée des compétences et ne remettent pas en cause, vu leur nature et leur portée, l'objectif d'une délimitation nette des compétences ${ }^{28}$. Dans la mesure où ces éléments sont explicitement mentionnés dans le texte du traité, on peut déterminer le champ des compétences transférées. Dès lors, le principe d'attribution reste effectivement en vigueur. Les règles qui encadrent l'exercice des compétences protègent aussi l'État membre. En effet, l'Union est tenue de respecter l'identité nationale des États membres ${ }^{29}$, le principe de coopération loyale ${ }^{30}$, le principe de

25 BverfG 30 juin 2009, 2 BvE 2/08, Lisbon, § 298.

26 Article 5.1 la phrase 1 et 5.2 TUE Lisbonne ; voir également l'article 1.1, article 3.6, article 4.1, l'article 48.6 (3) du traité UE de Lisbonne, l'article 2.1 et 2.2, article 4.1, article 7, l'article 19, article 32, article 130, l'article 132.1, l'article 207.6, l'article 337 TFUE ; déclaration $n^{\circ} 18$. relativement à la délimitation des compétences ; déclaration $\mathrm{n}^{\circ} 24$. concernant la personnalité juridique de l'Union européenne.

27 BverfG 30 juin 2009, 2 BvE 2/08, Lisbon, § 303 : La Cour évoque ici les compétences exclusives, les compétences partagées, les compétences pour des actions d'appui, de coordination ou de complément.

$28 \quad$ Ibidem, § 303.

29 Art. 4 § 2 TUE.

30 Art. 4 § 3 TUE. 
subsidiarité et de proportionnalité ${ }^{31}$. Ces règles renforcent le principe d'attribution et assignent un cadre précis et des limites à l'action de I'Union, ce qui conforte la souveraineté des États membres.

Au titre du second fondement, la Cour étudie les modalités de modification du droit de l'Union pour déterminer si une modification autonome est possible. Deux situations existent. Premièrement, certaines révisions du droit de l'Union supposent le consentement de l'État membre, alors la capacité d'autodétermination de l'État est conservée. Deuxièmement, certaines modifications du droit de l'Union peuvent intervenir sans que l'État n'ait à consentir et la Cour impose alors une obligation d'approbation nationale. Cette dernière situation n'affecte pas la constitutionnalité du traité de Lisbonne au regard de la Loi fondamentale mais a des incidences sur les Lois d'accompagnement qui doivent prévoir des procédures d'approbation dans le sens déterminé par la Cour. C'est ainsi qu'elle subordonne l'utilisation de la clause générale de transition de l'article $48 \S 7$ TUE à l'approbation préalable du Parlement national, par le biais d'une loi. Elle estime que la responsabilité pour l'intégration ne peut pas être assurée de façon complète ${ }^{32}$. La perte d'influence allemande doit être déterminée au moment de la ratification pour qu'elle puisse être légitimée démocratiquement. Or, tel n'est pas le cas avec ces procédures puisqu'il n'y a pas de certitude sur l'utilisation de ces clauses ni le domaine d'utilisation (bien que ce dernier soit déterminable). C'est pourquoi elle pose une exigence d'approbation parlementaire ${ }^{33}$. Cette attitude a été critiquée en doctrine car l'utilisation de la clause générale de transition peut être bloquée par un Parlement national. Ainsi, D. Doukas estime que la Cour ne fournit aucune explication valable sur le fait que le pouvoir de blocage du Parlement n'équivaut pas à une ratification formelle ${ }^{34}$. Cependant, l'obligation d'adopter une loi est plus contraignante car les pouvoirs publics constitutionnels sont tenus de prendre position sur l'utilisation de cette clause toutes les fois où l'occasion de se présentera ${ }^{35}$. Le pouvoir de véto en revanche suppose une implication

$31 \quad$ Art. 5 § 1,3 et 4 TUE.

32 BverfG 30 juin 2009, 2 BvE 2/08, Lisbon, § 317. Voir sur ce sujet, A. VON UNGERNSTERNBERG, "L'arrêt Lisbonne de la Cour constitutionnelle fédérale allemande, la fin de l'intégration européenne », RDP 1/2010, p. 175.

33 BverfG 30 juin 2009, 2 BvE 2/08, Lisbon, § 319. D. HANF, "L'encadrement constitutionnel de l'appartenance de l'Allemagne à l'Union européenne, l'apport de l'arrêt « Lisbonne » de la Cour constitutionnelle fédérale », CDE2009, n 5/6, p. 688.

34 D. DOUKAS, "The verdict of the German Federal Constitutional Court on the Lisbon Treaty : Not guilty, but don't do it again », ELRn ${ }^{\circ} 34,2009$, p. 875.

35 Pour une étude des conséquences de ces dispositions, lire X. MAGNON, précit., p. 420 . 
moindre " des responsables de l'intégration ». Il faut néanmoins convenir que dans les deux cas, l'Allemagne conserve la possibilité de décider de ce qui s'appliquera sur son territoire. Le pouvoir d'autodétermination pour la Cour suppose une volonté consciente et active et non une attitude passive.

Pour les clauses passerelles spécifiques, l'attitude est sensiblement identique. Pour la clause spéciale de transition conformément à l'article 81.3 (2) ${ }^{36}$ TFUE, la Cour exige une approbation parlementaire conformément à l'article 23.1 de la Loi fondamentale. Mais pour les autres clauses spéciales de transition, l'approbation parlementaire est toujours exigée mais la forme diffère. En effet, une loi n'est pas nécessaire, une décision du Bundestag et du Bundesrat dans la mesure où ses compétences sont affectées ${ }^{37}$ suffit. En effet, le droit de veto des parlements nationaux n'étant pas prévu, il fallait prévoir un mécanisme d'implication des organes législatifs allemands, au titre de la responsabilité pour l'intégration.

Pour la révision ordinaire des traités, la ratification selon les règles constitutionnelles est de mise. Ainsi, cette procédure sauvegarde la capacité d'autodétermination de l'Allemagne ${ }^{38}$.

La révision simplifiée de l'article 48 § 6 TUE suppose également une ratification selon les règles constitutionnelles, mais la Cour prend le soin de préciser que l'approbation parlementaire doit être identique à celle exigée pour la révision normale des traités 39 : une loi approuvée aux deux tiers par les membres du Bundestag et aux deux tiers des voix du Bundesrat ${ }^{40}$.

$\mathrm{Au}$ titre des dispositions permettant une modification du droit de l'Union, la Cour se penche sur l'article $311 \S 1$ TFUE relatif au budget et sur la clause de flexibilité de l'article 352 TFUE.

Ces deux procédures ne franchissent pas la "ligne rouge " 41 établie par la Cour et sauvegardent le pouvoir de décision de l'État, donc sa souveraineté. L'article $311 \S 1$ TFUE dispose que l'Union se dote des moyens nécessaires pour atteindre ses objectifs. La rédaction de l'article pouvait laisser croire à l'existence d'une marge de manœuvre. Mais la Cour ne l'interprète pas ainsi ${ }^{42}$, à bon droit car cette disposition s'utilise

BverfG 30 juin 2009, 2 BvE 2/08, Lisbon, § 319.

BverfG 30 juin 2009, 2 BvE 2/08, Lisbon, § 320, D. HANF, précit., p. 689.

Ibidem, § 307, 308.

Ibidem, § 312 .

C'est une jurisprudence constante, cf. D. HANF, précit., p. 687.

D. DOUKAS, précit., p. 875.

BverfG 30 juin 2009, 2 BvE 2/08, Lisbon, § 323, 324. 
uniquement dans le cadre des objectifs et des politiques de l'Union et ne peut servir à une extension de compétences.

En revanche, il en va différemment de la clause de flexibilité. La Cour l'analyse comme une clause qui assouplit le principe d'attribution ${ }^{43}$ et permet de modifier le traité et d'élargir les compétences de l'Union, et cela « en faisant abstraction du contexte historique de cette formulation, tout comme de l'évolution de l'utilisation de cette clause dans la pratique et la jurisprudence communautaire $"{ }^{44}$. Certes, il y a des garanties procédurales: l'unanimité au Conseil, l'approbation du Parlement, l'information des Parlements nationaux dans le cadre du respect du principe de subsidiarité. L'harmonisation des législations est exclue du champ d'application de cette clause. Mais la ratification n'est pas prévue par le traité. La Cour soumet donc l'utilisation de cette clause à une approbation parlementaire préalable ${ }^{45}$. Les garanties ne sont donc pas jugées suffisantes. Sur ce point, la Cour est particulièrement exigeante. En effet, elle considère comme insuffisants l'autorisation de ratification et le consentement du gouvernement fédéral démocratiquement élu ${ }^{46}$. D. Doukas estime que "cela dénote une profonde méfiance à l'égard du gouvernement fédéral et confie la primauté de la responsabilité pour l'intégration aux deux chambres et à la Cour constitutionnelle comme arbitre final ${ }^{47}$. D. Thym estime que cette hypothèse trouble la distinction entre l'adaptation du droit primaire et du droit dérivé ${ }^{48}$. Mais $\mathrm{J}$. Ziller propose une explication plus conciliante. Pour lui, " le but de ces procédures n'est pas de donner un droit de veto aux institutions allemandes, droit qui n'est pas prévu par le traité. Mais c'est seulement pour s'assurer de la conformité de la position allemande avec le vote de la majorité au Bundestag et au Bundesrat» 49. Pour d'autres en revanche, la procédure de ratification parlementaire rendra la clause de flexibilité inflexible 50 .

$43 \quad$ Ibidem, $\S 326$.

44 D. HANF, précit., p. 689

45 BverfG 30 juin 2009, 2 BvE 2/08, Lisbon, § 328.

46 D. DOUKAS, précit., p. 876. Nous traduisons.

47 D. DOUKAS, précit., p. 877.

48 D. THYM, "In the name of the sovereign statehood: a critical introduction to the Lisbon judgment of the German Constitutional court », CMLR December 2009, p. 1802.

49 J. ZILLER, "The German Constitutional Court's Friendliness towards European Law : On the Judgment of bundesverfassungsgericht over Ratification of the Treaty of Lisbon », European Public Law 16, no 1/2010, p. 53. Voir aussi p. 65, le point (3).

50 F. C. MAYER, "Rashomon à Karlsruhe », RTDE 46 (1), janv-mars 2010, p. 81, A. UNGERN-STERNBERG, précit., p. 182. 
En réalité, ces exigences ont une fonction importante : impliquer activement les pouvoirs publics allemands (et non le seul pouvoir exécutif) pour que l'intégration soit réalisée consciemment à chaque étape. C'est la conséquence de la responsabilité pour l'intégration. Cependant, la Cour retient une acceptation très exigeante de la souveraineté. En effet, prise sous cet angle, la conservation de la capacité d'autodétermination suppose l'expression de l'accord à chaque étape et non une fois pour toutes.

Toujours dans la dynamique du contrôle de la préservation de la souveraineté de l'État, la Cour prend en compte le droit de retrait. Ce dernier est le signe que le développement de l'Union ne va pas dans le sens de l'État fédéral, que l'intégration de l'Allemagne n'est pas irréversible; d'autant que le retrait est possible sans avoir à obtenir l'accord des autres États membres ${ }^{51}$. Cette possibilité fait que l'État est encore maître de son destin, donc souverain.

Quant au dernier point de contrôle, les requérants soulignaient que la primauté du droit de l'Union pouvait conduire à l'invalidation du droit constitutionnel contraire des États membres, que la généralisation des compétences de l'Union européenne rendrait en pratique impossible le contrôle du principe d'attribution limitées des compétences par la Cour constitutionnelle et qu'il ne lui serait plus possible de garantir l'identité constitutionnelle et la protection des droits fondamentaux ${ }^{52}$. La Cour rappelle donc son interprétation de la primauté et que les États membres ne peuvent être privés du droit d'examiner l'exécution du programme d'intégration ${ }^{53}$. Ainsi elle s'octroie le droit d'examiner si les instruments juridiques des institutions et organes européens restent dans les limites des pouvoirs souverains conférés, ou si la juridiction communautaire interprète les traités d'une manière extensive ${ }^{54}$.

Cette interprétation, pour critiquable qu'elle soit, n'en demeure pas moins inévitable. En effet d'une part, la Constitution ne peut qu'être la norme suprême dans un ordre juridique donné. Mais d'autre part la primauté du droit de l'Union est une nécessité pour la survie de ce droit. Cependant, les limites posées à la primauté sont les droits fondamentaux et l'identité constitutionnelle et en pratique, le conflit à ce niveau ne devrait pas survenir. II y va de la souveraineté des États membres de préserver ces éléments constitutionnels. Néanmoins, l'attitude de la Cour

51 BverfG 30 juin 2009, 2 BvE 2/08, Lisbon, § 329.

52 Ibidem, § 331, K. M. BAUER, "Conditions et contrôles constitutionnels de la validité interne du droit de l'Union », RTDE 45 (4), oct-déc. 2009, p. 815.

53 BverfG 30 juin 2009, 2 BvE 2/08, Lisbon, § 334.

54 Ibidem, $\S 338$. Pour une critique de cette attitude contradictoire, voir D. Doukas, précit., p. 879 et 880 . 
est moins conciliante et plus exigeante qu'elle ne l'a été lors de sa décision Maastricht. Mais la donne a changé puisque l'intégration est croissante. L'intérêt de cette décision est qu'elle insiste sur les points sensibles. La Cour joue pleinement son rôle de gardien de la Constitution en mettant en garde les pouvoirs publics constitutionnels sur un changement subreptice d'ordre constitutionnel. Rien n'est intangible mais les changements doivent être décidés démocratiquement et non de façon détournée.

L'examen de ces quatre éléments conforte la Cour dans l'idée que le traité ne porte pas une atteinte substantielle à la souveraineté de l'Allemagne.

La Cour tchèque était également invitée par le Président de la République à analyser l'atteinte à la capacité d'autodétermination. Elle y répond de façon indirecte en fixant sa conception de la souveraineté 55 . Elle évoque tout d'abord cette notion dans son sens d'indépendance par rapport à un pouvoir externe et de plus haut pouvoir sur le territoire de l'État. Cette indépendance signifie qu'aucune norme de droit international ne peut exister sans le consentement de l'État. Cependant, l'État doit exécuter les obligations auxquelles il a librement consenti, dans le respect du principe pacta sunt servanda. Ainsi, pour les juges tchèques, la possibilité de moduler ses compétences est surtout le signe d'une pleine souveraineté ${ }^{56}$. Jusque là, c'est une conception assez classique de la souveraineté. Et la Cour de poursuivre que la coopération internationale et la coordination des politiques nationales deviennent essentielles pour manager un monde global ${ }^{57}$. L'intégration communautaire peut en ce sens conduire in fine à une protection et un renforcement de la souveraineté ${ }^{58}$. Mais la Cour retient une acception concrète de la souveraineté, prise dans son sens de puissance non subordonnée à un pouvoir externe. Pour les juges tchèques, d'autres formes de pouvoirs très puissants émergent à côté de l'État. Ainsi, l'exercice en commun de certaines compétences renforcera la puissance concrète de l'État face à ces pouvoirs émergents. On peut noter une sorte de confusion. Si puissants que ces pouvoirs puissent être, ils ne seront jamais souverains, au sens où ce terme est entendu traditionnellement en doctrine. La souveraineté comprise dans son sens de puissance est surtout une puissance légitime. Or, ces pouvoirs émergents n'ont pas la légitimité de l'État.

55 Décision du 26 novembre 2008, aff. PI. ÚS n 19/08, pt 98 à 100.

56 Ibidem, pt 100.

57 Ibidem, pt 101.

58 Ibidem, pt 102. 
En revanche, la Cour est extrêmement lucide sur les conséquences de la mondialisation en terme de gestion des compétences étatiques 59 et met en avant le concept de pooled sovereignty ${ }^{60}$, que l'on peut traduire par souveraineté de groupe. II doit sans doute s'agir de l'exercice partagé des compétences étatiques. Cette gestion en commun de certaines compétences est compatible avec l'indépendance de l'État, notamment parce que les États conservent un droit de retrait de l'Union 61 .

L'importance du droit de retrait n'apparaît pas dans la décision française. Mais le Conseil constitutionnel avait déjà souligné l'importance de cette faculté dans sa jurisprudence antérieure ${ }^{62}$. Le droit de retrait est un élément garant de la souveraineté de l'État car c'est le signe qu'il a le dernier mot.

Sur ce dernier point, les juges français et tchèque rejoignent leurs homologues allemands. Mais seulement sur ce point, car là où les juges allemands tentent de conserver un espace suffisant de décision aux organes exécutifs et législatifs allemands au nom de la responsabilité pour l'intégration, les juges tchèques voient dans l'exercice en commun des compétences un renforcement de la souveraineté ${ }^{63}$. Mais la Cour constitutionnelle tchèque fait la part belle à l'examen des compétences transférées. C'est d'ailleurs le cœur des trois décisions.

\section{B - L'évaluation du maintien de la souveraineté étatique à l'aune des transferts de compétence}

La souveraineté est surtout appréhendée par les cours constitutionnelles dans son acception matérielle en raison de la nature de l'impact du traité. En effet, le traité de Lisbonne opère des transferts de compétences dans deux dimensions. D'une part, certaines compétences qui appartenaient à l'État sont désormais transférées à l'Union; d'autre part, les modalités d'exercice de certaines compétences appartenant déjà à l'Union sont modifiées dans le sens où l'État perd un pouvoir d'influence. L'ampleur des transferts a-t-il un impact sur la souveraineté des États membres? Cette question, posée à toutes les juridictions constitutionnelles, a suscité des réponses différentes. En France, le

$59 \quad$ Ibidem, pt 105.

$60 \quad$ Ibidem, pt 104.

61 Ibidem, pt 106.

62 CC, 85-188 DC, 22 mai 1985, Protocole $\mathrm{n}^{\circ} 6$ additionnel à la Convention européenne de sauvegarde des Droits de l'homme et des libertés fondamentales concernant l'abolition de la peine de mort, signé par la France le 28 avril 1983, pt. 1.

63 Décision du 26 novembre 2008, aff. PI. ÚS n 19/08, pt 108 et 109. 
Conseil constitutionnel a relevé des atteintes aux conditions essentielles d'exercice de la souveraineté mais la révision constitutionnelle neutralise les inconstitutionnalités pour permettre la ratification du traité. En Allemagne, la Cour constitutionnelle fédérale a déclaré qu'il n'y avait pas de violation de la Loi fondamentale mais fait peser sur les lois d'accompagnement des obligations d'implication et de contrôle des affaires européennes rendant ainsi possible la ratification du traité. En République tchèque, la Cour constitutionnelle estime que la souveraineté est préservée et que le traité ne porte pas atteinte à l'ordre constitutionnel, ouvrant donc la voie à la ratification.

Les juges constitutionnels posent deux séries de conditions de constitutionnalité des transferts de compétences. La première condition matérielle est relative à la nature de la compétence transférée. La seconde condition formelle porte sur les modalités du transfert.

\section{1) L'examen de la nature des transferts}

Tous les juges constitutionnels examinent la nature de la compétence transférée, ce qui recouvre le contenu de la compétence transférée mais également l'intensité du transfert.

La souveraineté prise dans son sens matériel correspond à l'idée qu'elle repose sur certains droits de puissance. La dépossession de certaines compétences confine à l'anéantissement de la souveraineté. $\mathrm{Si}$ cette dernière se conçoit dans son sens premier comme le plus haut pouvoir que l'on rencontre sur un territoire donné, encore faut-il que le détenteur de ce pouvoir puisse l'exercer matériellement. En ce sens, l'acception première de la souveraineté rejoint son acception matérielle.

\section{a) Le contenu des transferts}

Les juges constitutionnels ont été amenés, par les requérants, à contrôler si l'ampleur des transferts ne portaient pas atteinte à la souveraineté de l'État. Cela suppose en premier lieu d'accepter l'idée qu'il existe une limite au transfert de compétences et en second lieu une définition concrète de cette limite, ce qui pose en amont le problème de l'autorité légitime pour dessiner une telle frontière.

- La reconnaissance implicite d'une limite aux transferts de compétences

Les juges constitutionnels sont tous d'accord sur le principe de l'existence d'une telle limite. La Constitution tchèque autorise, en son 
article 10 a certains transferts de compétences. Cet article doit être interprété en relation avec d'autres dispositions constitutionnelles: l'article $1 \S 1$ et $9 \S 2$. Ainsi, les transferts de pouvoirs ne peuvent aller jusqu'à violer l'essence même de la République, comprise comme un État démocratique et souverain gouverné par l'État de droit, fondé sur le respect des droits et libertés de l'être humain et des citoyens, ni jusqu'à changer les exigences essentielles d'un État démocratique gouverné par l'État de droit ${ }^{64}$. La Cour en déduit qu'au niveau formel, le transfert de pouvoir est limité par la préservation des fondations de la souveraineté étatique de la République tchèque de l'article $1 \S 1$ de la Constitution ${ }^{65}$. Pour autant, cela ne signifie pas que la compatibilité du transfert soit subordonnée à la rétention par la République tchèque de certains pouvoirs 66 .

Le juge allemand pose aussi expressément une limite aux transferts de compétences ${ }^{67}$ : un noyau dur de compétences doit rester à l'État, " a sufficient space for the political formation of the economic, cultural and social circumstances of life » ${ }^{68}$, sous peine de perdre son caractère souverain 69 .

Pour le Conseil constitutionnel, la Constitution française pose également une limite aux transferts de pouvoirs. Mais cette limite est en réalité déduite de la constatation de l'atteinte aux conditions essentielles d'exercice de la souveraineté ${ }^{70}$.

$\mathrm{Si}$ les trois juges constitutionnels se rencontrent sur le concept d'une limitation matérielle des transferts de pouvoirs souverains, la substance de cette limite n'est pas la même. Autant les juges allemand et français sont capables de déterminer une liste de compétences dont le transfert porte atteinte à la souveraineté, autant le juge tchèque refuse de raisonner en terme de "liste », puisque le transfert ne doit pas porter atteinte au caractère démocratique et souverain. II existe bien un material core, mais il ne trouve pas à s'exprimer en terme de compétences. Dans la seconde décision relative au traité de Lisbonne, la Cour, invitée par le

64 Décision du 26 novembre 2008, aff. PI. ÚS n 19/08, pt 97.

65 Ibidem, pt 130. La condition matérielle de constitutionnalité du transfert est relative à la manière dont sont exercés les pouvoirs conférés, limitation de l'article $9 \S 2$ de la Constitution.

66 Décision du 26 novembre 2008, aff. PI. ÚS nº 19/08, pt 130.

67 BverfG 30 juin 2009, 2 BvE 2/08, Lisbon, § 226.

68 BverfG 30 juin 2009, 2 BvE 2/08, Lisbon, § 249, A. VON UNGERN-STERNBERG, précit., p. 178.

69 X. MAGNON, précit., p. 435.

70 CC, 560 DC, 20 décembre 2007, Traité modifiant le traité sur l'Union européenne et le traité instituant la Communauté européenne, pt 18. Le juge français n'est aussi dogmatique que ses homologues. 
Président de la République ${ }^{71}$, a expressément refusé de créer un catalogue de droits non transférables et de façonner autoritairement une limite substantielle au transfert de pouvoirs ${ }^{72}$. Elle préfère une méthode casuistique pour des raisons de minimalisme judiciaire ${ }^{73}$, déjà mise en œuvre au demeurant ${ }^{74}$, et renvoie au pouvoir politique une telle définition 75 .

Cette attitude tranche singulièrement avec celle des juges allemand et français, qui s'estiment compétents. Le noyau de compétences non transférables ne peut être inscrit que dans la Constitution. En tant que fondement de l'ordre juridique, la Constitution est naturellement le texte qui pose les limites à ne pas transgresser pour que l'État reste souverain et démocratique. Ainsi, le juge constitutionnel, interprète autorisé de la Constitution, est le mieux placé pour effectuer ce travail de définition, ce travail d'interprétation de la Constitution. Or pour le juge tchèque, cette question est plutôt d'essence politique ${ }^{76}$. L'attitude de la Cour n'est pas exempte d'ambigüité. D'un côté, elle renvoie au pouvoir législatif la tâche de déterminer ce qui relève de la souveraineté étatique, de l'autre, elle accepte ce travail de définition mais seulement au cas par cas. Établir un catalogue fige la situation et peut freiner l'intégration européenne ultérieure. Tel est l'effet induit de la position des juges constitutionnels allemands ${ }^{77}$. Les juges tchèques ont préféré une hypothèse plus souple, d'autant qu'en s'en remettant au pouvoir politique, l'entreprise était également renvoyée aux calendes grecques. On imagine mal un projet de loi constitutionnelle portant sur la définition de tels éléments. Pourtant, protéger la souveraineté étatique suppose qu'on définisse clairement quelles sont les compétences dont le transfert porterait atteinte au caractère souverain de l'État. Le problème dans la décision tchèque est que les juges ont une motivation allusive. Ils affirment la constitutionnalité des transferts plus qu'ils ne l'expliquent. En réalité, la Cour est très favorable au concept de pooled sovereignty, ce qui l'empêche conceptuellement de poser des limites matérielles. Mené à son terme, un tel raisonnement révèle ses faiblesses. Un État qui n'a plus de domaines de décision propre est-il encore souverain? II est concevable de transférer nominativement la majeure partie des compétences étatiques tout en conservant formellement à l'État, la

Décision du 3 novembre 2009, aff. PI ÚS n²9/09, pt 110.

Ibidem, pt 111 et 112 .

Ibidem, pt 113.

Ibidem, pt 113.

Ibidem, pt 111.

Ibidem, pt 111.

D. HANF, précit., p. 684 
compétence de la compétence. Est-ce que pour autant un tel État resterait souverain?

La position française est encore différente. Le juge français fait pleinement son travail d'interprétation de la Constitution en définissant ce qui est une matière inhérente à l'exercice de la souveraineté nationale. Cependant, dans le système français, le problème provient plutôt de ce que l'inconstitutionnalité peut être surmontée par une nouvelle loi constitutionnelle dont le juge refuse le contrôle. En effet, dans sa décision du 2 septembre 1992, le Conseil constitutionnel a affirmé que le " pouvoir constituant est souverain» et donc qu'il «lui est loisible d'abroger, de modifier ou de compléter les dispositions de valeur constitutionnelle " ${ }^{78}$. Comment faire respecter les limites sans contrôle des lois constitutionnelles? In fine, on pourrait changer d'État sans que le Conseil ne puisse le sanctionner 79 .

L'absence de contrôle est une situation inenvisageable en Allemagne. La Cour prend soin de préciser que l'avènement d'un ordre juridique nouveau supposerait l'abolition de la Loi fondamentale actuelle et une décision du peuple souverain ${ }^{80}$. L'article 146 de la Loi fondamentale définit la limite à l'intégration européenne, et cette limite ne peut être franchie que par le pouvoir constituant et non par les autorités de l'État qui sont fondées sur la Constitution ${ }^{81}$. De plus, "la Cour déclare sa juridiction sur les transitions constitutionnelles, en garantissant à chaque citoyen le droit de se plaindre à la Cour sur l'adoption nécessaire d'une nouvelle Constitution " ${ }^{82}$. D'après $D$. Thym, la décision Lisbonne pourrait servir de fondement à des référendums constitutionnels pour les modifications ultérieures des traités, même s'il concède que la nécessité d'un référendum n'est pas évidente en soi. II précise que la Cour n'utilise pas ce terme, d'autres moyens d'exprimer la volonté populaire sont en effet concevables ${ }^{83}$.

La multiplicité des solutions retenues pour régler un problème identique révèle la difficulté à trouver la bonne solution. La décision allemande est fortement critiquée en raison de son caractère défavorable

78 CC, 2 septembre 1992, 92-312 DC, Maastricht II, pt. 19. Cette position a été réitérée dans la décision 496 DC du 26 mars 2003, pt 3.

79 Sur la difficulté de déterminer le seuil critique où la souveraineté est atteinte: K. BECHET-GOLOVKO, précit., p. 82.

80 BverfG 30 juin 2009, 2 BvE 2/08, Lisbon, § 346.

81 BverfG 30 juin 2009, 2 BvE 2/08, Lisbon, § 179, F. SCHORKOPF, « The European Union as an association of sovereing states, Karlsruhe's ruling on the Treaty of Lisbon », The german Law Journal, vol. 10, n 8, p. 1238.

82 D. THYM, précit., p. 1803, D. DOUKAS, précit., p. 873. BverfG 30 juin 2009, 2 BvE 2/08, Lisbon, § 218.

83 D. THYM, précit., p. 1803. 
à la construction européenne, la décision tchèque est critiquée dans son propre pays pour laxisme ${ }^{84}$, la décision française n'est pas non plus exempte de critiques car elle ne pose pas de limites ${ }^{85}$ à la construction européenne. Malgré la diversité des ordres juridiques, le traité de Lisbonne pose pour tous le problème de la souveraineté des États membres. Or, il y a un paradoxe à penser avec des critères étatiques un objet qui se définit comme sui generis. L'État est un cadre de réflexion indépassable et pourtant il n'est pas pertinent en tous points. De plus, comment expliquer que les mêmes transferts de compétences portent atteinte à la souveraineté d'un État (français) mais pas d'un autre (tchèque) ?

Les mêmes dissemblances se retrouvent lorsque l'on examine le contenu des compétences dites de souveraineté.

- Le contenu de la limite : la liste des matières de souveraineté

Une fois posé le principe d'une limite aux transferts, encore faut-il déterminer précisément ce qui ne peut être transféré.

Les juges allemands estiment que l'État doit conserver un espace de décision suffisant dans des domaines clés: la citoyenneté, le monopole de la violence légitime, le droit pénal ${ }^{86}$, les revenus et dépenses de l'État, l'engagement du crédit, les recettes et dépenses, la langue, le droit de la famille et de l'éducation, la liberté d'opinion, de presse et d'association et les questions liées au statut des confessions religieuses ${ }^{87}$. Cette liste a été vivement critiquée en doctrine. La Cour ne convainc pas ${ }^{88}$. Pour $\mathrm{C}$. Schönberger, ce sont des affirmations gratuites et souvent vagues ${ }^{89}$. Le principal reproche tient à ce que la liste des pouvoirs législatifs devant être réservés à l'État membre coïncide étrangement la liste des pouvoirs restant actuellement aux États membres ${ }^{90}$. On pourrait être d'accord sur cette liste mais battre la

84 Le second recours témoigne de l'hostilité des pouvoirs publics à l'égard de l'attitude compréhensive des juges tchèques vis-à-vis du traité de Lisbonne. Voir également P. BRIZA, «The Czech Constitutional Court on the Lisbon Treaty », European constitutional law review 2009, v. 5, $\mathrm{n}^{\circ} 1$, p. 143.

85 CPJI, 17 août 1923, Wimbledon, Série A, n¹, p. 24.

86 Pour une analyse des questions de droit pénal, voir $\mathrm{F}$. JESSBERGER, K. KRETSCHERMER, L'arrêt de la Cour constitutionnelle allemande du 30 juin 2009 Les implications du traité de Lisbonne sur le droit pénal européen, RSC 2010, p. 111.

87 BverfG 30 juin 2009, 2 BvE 2/08, Lisbon, § 249, 252 à 260.

88 A. VON UNGERN-STERNBERG, précit., p. 179.

89 C. SCHÖNBERGER, «Lisbon in Karlsruhe: Maastricht's Epigones at Sea », The German Law Journal, vol. $10 \mathrm{n}^{\circ}$ 8, p. 1209.

90 C. SchÖnBERgeR, précit., p. 1209 ; D. ThYM, précit., p. 1801 ; D. HANF, précit., p. 684 ; F. C. MAYER, précit., p. 91. 
monnaie est indéniablement une marque de souveraineté et pourtant une telle compétence a déjà été transférée ${ }^{91}$. Ainsi, pour $C$. Schönberger, la description des pouvoirs réservés est ainsi de pure opportunité politique et non une interprétation de principes constitutionnels ${ }^{92}$. Et il est difficile de ne pas y souscrire. Si dans le principe, il est nécessaire de conserver des zones de décisions à l'État, il est relativement complexe d'en définir le contenu.

En réalité, seule une compétence ne peut être transférée: la compétence de la compétence, le pouvoir ultime. La Cour constitutionnelle allemande l'a bien souligné ${ }^{93}$. D'autant que l'État ne transfère des compétences que parce qu'il est souverain; ce faisant il exerce sa souveraineté. Suivant l'intensité du transfert, il choisit de se soumettre à la volonté de la majorité. Lorsqu'ultérieurement une décision est prise et que l'État en question n'est pas d'accord avec cette décision, l'application de cette dernière ne se fait pas en violation de sa souveraineté, puisqu'il a accepté cette éventualité. En conservant la compétence de la compétence, l'État refuse à l'Union cette autonomie signe de souveraineté et a toujours la possibilité de reprendre ce qu'il a donné.

Les juges tchèques raisonnent différemment. II n'est pas possible de transférer des compétences qui portent atteinte à la souveraineté et l'unité de la République ${ }^{94}$, mais la Cour refuse de définir concrètement quelles sont ces compétences ${ }^{95}$. De façon surprenante, elle rajoute que le respect de la souveraineté n'est pas subordonné à la rétention par la République tchèque de certains pouvoirs. À chaque fois que le requérant demande à la Cour de déterminer si le transfert d'une compétence est compatible avec l'article 10a, elle répond par l'affirmative. Selon les requérants, l'article 216 TFUE affecte la capacité internationale du Président et du Parlement tchèque qui n'aurait pas l'occasion d'exprimer son consentement à un traité. De plus, il ne serait pas possible d'examiner la constitutionnalité de ce traité. En conséquence, le Sénat demande à la Cour s'il est possible d'appliquer ces traités sur la base de l'article 10a qui autorise seulement certains transferts de pouvoirs. Pour la Cour, cet article n'affecte pas la négociation des traités conclus par I'Union, ni leur application dans l'ordre constitutionnel tchèque ${ }^{96}$. Lors de l'examen de la compatibilité de l'article 216 TFUE, la Cour rappelle que le

91 F. C. MAYER, précit., p. 91 ; C. SCHÖNBERGER, précit., p. 1209.

92 C. SCHÖNBERGER, précit., p. 1209.

93 BverfG 30 juin 2009, 2 BvE 2/08, Lisbon, § 233.

94 Décision du 26 novembre 2008, aff. PI. ÚS n 19/08, pt 109.

95 Ibidem, pt 109.

96 Ibidem, pt 182. 
transfert est limité par la préservation des principaux attributs d'un État souverain ${ }^{97}$. En l'espèce, une telle frontière n'est pas dépassée dès lors que les organes de l'Union n'excèdent leurs compétences ${ }^{98}$. Ainsi, l'important pour les juges tchèques ne réside pas dans la nature de la compétence transférée mais dans le respect des conditions du transfert ${ }^{99}$. Ce n'est pas un problème de fond mais un problème de forme.

Dans la décision Lisbonne II, la question des compétences non transférables a été posée à nouveau. Cette fois-ci, le requérant estimait que la défense était un pouvoir devant demeurer à l'État souverain ${ }^{100}$. Ainsi, la création d'une défense européenne commune serait inconstitutionnelle. La Cour estime que la défense n'est pas vraiment une marque de souveraineté et que la création d'un système interétatique de défense collective ne viole en aucune façon la souveraineté des États qui partagent ce système. Étant donné que la République tchèque fait déjà partie de I'OTAN, l'argument des requérants manque de cohérence ${ }^{101}$. Sur ce dernier point, on ne peut qu'être d'accord avec les juges constitutionnels tchèques. Et pourtant, s'il y a une compétence qui ressemblait à une marque de souveraineté 102 c'est bien la défense. Cette réponse illustre bien à quel point les juges tchèques refusent de réfléchir en terme de compétences de souveraineté. La citoyenneté, dont les requérants estimaient que l'introduction serait contraire à la souveraineté, a suscité une réponse similaire, d'autant qu'il est un peu tard à l'heure de Lisbonne, de soulever l'inconstitutionnalité d'une citoyenneté qui existe depuis le traité de Maastricht ${ }^{103}$. La citoyenneté 104 de l'Union ne nie pas celle des États membres mais l'enrichit d'une dimension européenne et en ce sens, elle n'est pas inconstitutionnelle. La décision Lisbonne II confirme que pour les juges tchèques la souveraineté ne réside pas dans certaines compétences, mais dans le respect des conditions de leur transfert. En revanche, comme les juges allemands, ils soulignent que la compétence de la

\footnotetext{
97 Ibidem, pt 184.

98 Ibidem, pt 184

99 P. BRIZA, précit., p. 158.

100 Décision du 3 novembre 2009, aff. PI ÚS n²9/09, pt 145.

101 Décision du 3 novembre 2009, aff. PI ÚS n 29/09, pt 152.

102 R. CARRE DE MALBERG, op. cit., p. 76.

103 Décision du 3 novembre 2009, aff. PI ÚS n 29/09, pt 160.

104 A ce sujet, voir J. HEYMANN, « De la citoyenneté de l'Union comme révélateur de la nature de l'Union européenne (à propos de l'arrêt Rottmann) ", Europe $n^{\circ} 6$ Juin 2010, Étude 7.
} 
compétence reste aux États membres, ce qui conforte la souveraineté de la République tchèque ${ }^{105}$.

À l'opposé, il existe clairement des matières de souveraineté pour le juge français. En effet, les articles relatifs à la lutte contre le terrorisme, au contrôle aux frontières, à la lutte contre la traite des êtres humains, à la coopération judiciaire en matière civile et en matière pénale, au Parquet européen portent atteinte aux conditions essentielles d'exercice de la souveraineté nationale ${ }^{106}$. Le juge prend en compte le critère matériel ${ }^{107}$, le « caractère régalien » des compétences transférées ${ }^{108}$. II ne peut qu'en déduire une atteinte à la souveraineté ${ }^{109}$. II n'échappe à personne que les traités communautaires amputent l'État de compétences de plus en plus importantes, jusqu'à toucher le noyau des compétences régaliennes. La Constitution est régulièrement révisée pour neutraliser de telles atteintes. Le juge constitutionnel français pose une limite aux transferts mais cette dernière peut être surmontée. Au fil des révisions, que reste-il de cette souveraineté ? ${ }^{110}$. Le juge français refuse de faire une analyse cumulée des transferts. Même si aucune limite n'est intangible car tout État peut en dernière instance accepter de perdre sa souveraineté pour se fondre dans un État fédéral, le juge constitutionnel français reste en deçà de ses homologues en refusant de poser une limite aux atteintes à la souveraineté dans le cadre de la Vème République.

Ainsi, pour apprécier l'atteinte à la souveraineté, les juges constitutionnels limitent matériellement les transferts de compétences à des degrés divers. Le critère matériel n'est pas le seul à entrer en ligne de compte. Il est couplé à un critère que l'on peut qualifier de formel, lié à l'intensité des transferts.

105 Décision du 26 novembre 2008, aff. Pl. ÚS n 19/08, pt 132.

106 CC, 560 DC, 20 décembre 2007, Traité modifiant le traité sur l'Union européenne et le traité instituant la Communauté européenne, pt 18 et 19.

107 Qui est combiné avec un critère formel et temporel : F. CHALTIEL, " La décision du Conseil constitutionnel relative au Traité de Lisbonne : Autorité de la chose jugée et contribution à la définition de l'Union », LPA 4 janvier $2008, n^{\circ} 4, p .8$.

108 B. MATHIEU, «Le Traité de Lisbonne et la Constitution, ou comment régler le passif du «traité constitutionnel ? », JCP Ed. G. $n^{\circ} 17,13$ février 2008, I 116, pt 10 ; M. Guillaume, « La Constitution et le traité de Lisbonne du 13 décembre 2007 (Cons. Const., 20 décembre 2007) », LPA 25-26 décembre 2007 n² 257-258, p. 3.

109 Voir à ce propos, O. GOHIN, « Conseil constitutionnel et Traité de Lisbonne : vite, clair et bien », JCPA n 3, 14 janvier 2008, p. 20.

110 O. GoHIN, précit., p. 22. 


\section{b) L'intensité des transferts}

Un transfert de compétences est susceptible de degrés. La variation est fonction du mode de décision. Si l'unanimité est requise, l'État conserve un droit de veto. Si la décision est prise selon une majorité qualifiée ou autre, l'État perd un pouvoir d'influence. Ainsi l'intensité des transferts doit entrer en ligne de compte dans l'appréciation de l'affectation de la souveraineté ${ }^{111}$. L'intensité est également liée au jeu des principes de subsidiarité (article 5.3 TUE) et de proportionnalité (article 5.4 TUE). En tant qu'ils encadrent l'utilisation par les organes de l'Union des compétences transférées, ces principes sont pris en compte dans l'examen de l'atteinte à la souveraineté.

- L'impact des principes de subsidiarité et de proportionnalité

À propos du principe de subsidiarité, le juge constitutionnel français estime que "la mise en œuvre de ce principe pourrait ne pas suffire à empêcher que les transferts de compétence autorisés par le traité revêtent une ampleur ou interviennent selon des modalités telles que puissent être affectées les conditions essentielles d'exercice de la souveraineté nationale » ${ }^{112}$. En effet, " la subsidiarité constitue en effet moins une règle de répartition des compétences entre l'Union et les États membres qu'un principe permettant de moduler l'intensité de l'intervention de I'Union par rapport à celle des États dans les seules compétences partagées " ${ }^{113}$. Ainsi, ce principe n'est en " aucun cas un facteur d'atténuation ni d'exonération d'une atteinte à la souveraineté »114. Pourtant, ce principe est renforcé : il est désormais justiciable, il s'étend au second et troisième pilier par l'effet de la fusion, le comité des régions et les parlements nationaux ont vocation à veiller à

111 M. GAUTIER, « La décision du Conseil constitutionnel relative au traité établissant une Constitution pour l'Europe est-elle une grande décision », JCP $A \mathrm{n}^{\circ}$ 3, 17 Janvier 2005, 1025.

112 CC, 560 DC, 20 décembre 2007, Traité modifiant le traité sur l'Union européenne et le traité instituant la Communauté européenne, pt 16. C'est une jurisprudence constante depuis 1997.

113 O. DORD, « Le Conseil constitutionnel face à la « Constitution européenne » : contrôle des apparences ou apparence de contrôle ? ", AJDA 2005 p. 215.

114 F. ChALTIEL, précit., p. 9. Voir aussi, H. LABAYLE, "La Constitution française à l'épreuve de la Constitution pour l'Europe », RFDA 2005 p. 15; A. LEVADE, «Le Conseil constitutionnel aux prises avec la Constitution européenne ", RDP 1/2005, p. 43 ; D. SIMON, «L'examen par le Conseil constitutionnel du traité portant établissement d'une Constitution pour l'Europe : fausses surprises et vraies confirmations », Europe n² 2, février 2005, Etude 2, pt 21. 
son respect. Le Conseil constitutionnel refuse d'exagérer la vertu protectrice de ce principe ${ }^{115}$.

Pour les juges tchèques, les principes de subsidiarité et de proportionnalité fournissent un cadre normatif suffisant pour déterminer la portée du transfert de pouvoirs ${ }^{116}$. L'analyse de ces principes prend place dans une analyse plus globale des conditions de constitutionnalité des transferts. Ces principes participent à la «déterminabilité » des transferts de compétence. De plus, leur respect pouvant être obtenu par la voie juridictionnelle, elle considère que le cadre institutionnel européen permet un contrôle adéquat du respect des limites des transferts. Le juge tchèque est en réalité beaucoup plus confiant que le juge français.

Le juge allemand analyse le principe de subsidiarité au titre des mécanismes de protection de l'exercice des compétences des États membres. II note l'existence de mécanismes pour garantir ce principe mais lucide, il explique que l'effectivité dépendra en grande partie de la mesure dans laquelle les parlements nationaux pourront prendre des dispositions organisationnelles qui les placent en position de faire un usage approprié du mécanisme dans la courte période de huit semaines ${ }^{117}$.

Si l'analyse de ce même principe est différente selon les juges, on remarque qu'il n'a globalement suscité que peu de développements, les juges étant très réalistes au fond sur sa portée.

Ainsi, les principes de subsidiarité et de proportionnalité ne jouent que de façon marginale sur l'intensité du transfert et, in fine, sur la protection de la souveraineté.

L'examen de l'atteinte à la souveraineté passe également par celui de l'étendue de la compétence transférée.

- L'impact de changement du mode d'exercice des compétences

Le changement du mode d'exercice des compétences peut affecter la souveraineté d'un État. Seul le Conseil constitutionnel le prend en compte ${ }^{118}$.

On peut donc distinguer trois situations. D'une part, la perte du droit de veto dans une matière régalienne implique que la France puisse être obligée par une majorité d'autres États membres à un acte contraire à ses vues et ou à ses intérêts. Évidemment, l'atteinte à la souveraineté

115 J. Roux, «Le traité établissant une Constitution pour l'Europe à l'épreuve de la Constitution française », RDP 1/2005, p. 90.

116 Décision du 26 novembre 2008, aff. Pl. ÚS nº 19/08, pt 137.

117 BverfG 30 juin 2009, 2 BvE 2/08, Lisbon, § 305.

118 CC, 560 DC, 20 décembre 2007, Traité modifiant le traité sur l'Union européenne et le traité instituant la Communauté européenne, pt 20. 
est plutôt théorique et « procède de la simple possibilité que la nation soit contrainte dans un domaine régalien " ${ }^{119}$. La perte du droit de veto se produit lors du passage de l'unanimité à la majorité qualifiée (article 31.2 TUE, 82, 83, 85, 87 à 89, 133 et 329 TFUE) ${ }^{120}$. Cette jurisprudence est constante depuis la décision relative au traité de Maastricht ${ }^{121}$.

D'autre part, l'octroi d'un pouvoir de décisionnel au Parlement européen qui n'est pas l'émanation de la souveraineté nationale porte atteinte à cette dernière. Ainsi, l'article 218.6 a) v) TFUE qui étend le domaine des accords dont le Conseil ne peut approuver la conclusion qu'après l'approbation du Parlement européen implique une révision de la Constitution ${ }^{122}$. "La position du Conseil constitutionnel est fixée depuis sa décision du 30 décembre 1976 : il « n'appartient pas à l'ordre institutionnel de la République française et (...) ne participe pas à l'exercice de la souveraineté nationale ». Dès lors, les stipulations, [...] lui conférant une fonction décisionnelle appellent une révision de la Constitution, en ce qu'elles lui confient un rôle normatif et privent les autorités nationales des pouvoirs correspondants $» 123$.

Enfin, le Conseil constitutionnel confirme que la perte du pouvoir d'initiative constitue également une atteinte aux conditions essentielles d'exercice de la souveraineté nationale. II s'agit ici de l'article 76 TFUE qui permet l'adoption d'actes relatifs à la coopération administrative sur proposition de la Commission ou d'un quart des États membres. Cette confirmation est bienvenue car la perte du pouvoir d'initiative "semblait jusqu'alors considérée comme un simple élément subsidiaire, insusceptible, à lui seul, de conduire à la méconnaissance de la souveraineté étatique dans ses conditions essentielles d'exercice » 124

Le Conseil constitutionnel réalise dans cette décision un effort pédagogique de détermination des conditions de l'atteinte à la souveraineté. Les autres juges ont réalisé un travail similaire mais dans leur démarche l'intensité du transfert n'entre pas en ligne de compte. Ils ont posé d'autres conditions de constitutionnalité des transferts de compétence, fonction des modalités du transfert.

119 J.-E. SCHOETTL, " La ratification du "Traité établissant une Constitution pour l'Europe" appelle-t-elle une révision de la Constitution française ? ", LPA, 29 novembre 2004 (238), p. 9.

120 CC, 560 DC, 20 décembre 2007, Traité modifiant le traité sur l'Union européenne et le traité instituant la Communauté européenne, pt 21.

121 A. LeVADE, précit, p. 45.

122 CC, 560 DC, 20 décembre 2007, Traité modifiant le traité sur l'Union européenne et le traité instituant la Communauté européenne, pt 22.

123 A. LEVADE, précit, p. 45.

124 J. RouX, précit., p. 93. 


\section{2) L'étude des modalités du transfert}

À la lecture des décisions rendues par les juridictions constitutionnelles, on constate que la compatibilité entre souveraineté et transferts de compétences est fonction de la nature de la compétence et des modalités du transfert. En effet, les juges ont posé des conditions formelles à la constitutionnalité des transferts. Un blanc-seing n'est pas en général considéré comme constitutionnel car le respect de la souveraineté suppose que soit déterminée avec plus ou moins de précision l'étendue de la compétence abandonnée.

Sur ce point, les décisions ne sont pas uniformes. Le juge français ne raisonne pas en ces termes, mais les juges allemand et tchèque subordonnent la constitutionnalité des transferts au respect de certaines conditions liées aux modalités des transferts. Une condition est commune à ces deux ordres constitutionnels: les transferts doivent être précis, déterminables, révocables ${ }^{125}$.

Les autres sont propres à chaque constitution. Pour les juges tchèques, le transfert doit être effectué souverainement ${ }^{126}$. Cependant, les requérants n'ont pas donné l'occasion à la Cour d'appliquer cette condition.

Les juges allemands conditionnent la constitutionnalité des transferts au respect du principe d'attribution et de l'identité constitutionnelle ${ }^{127}$. Le principe d'attribution est respecté si les transferts sont précis et déterminables car c'est à cette seule condition que l'on peut identifier le programme d'intégration. S'il est impossible à identifier, alors l'État donne un blanc seing à l'Union et lui cède la compétence de la compétence. Donc ce critère n'appelle pas de réflexion autre que celle menée dans le cadre de la condition commune de constitutionnalité des transferts. L'identité constitutionnelle renvoie essentiellement dans la décision Lisbonne au principe démocratique. Cette condition du transfert sera donc examinée dans la partie relative au respect par le traité de Lisbonne de la démocratie. II faut rappeler que l'étroite imbrication des éléments, surtout dans l'arrêt allemand, rend difficile leur scission, qui est pourtant nécessaire pour mener à bien cette étude.

Dans les décisions tchèque et allemande, on peut relever d'une part l'affirmation très pédagogique des conditions de constitutionnalité

125 Décision du 26 novembre 2008, aff. PI. ÚS n 19/08, pt 130 et BverfG 30 juin 2009, 2 BvE 2/08, Lisbon, § 233.

126 Décision du 26 novembre 2008, aff. Pl. ÚS n 19/08, pt 108.

127 BverfG 30 juin 2009, 2 BvE 2/08, Lisbon, § 226. 
des transferts, d'autre part la confrontation des dispositions du traité à ces conditions.

\section{a) Des transferts précis, déterminables et révocables}

La souveraineté peut se concilier avec des transferts continus de compétence. Mais pour les juges, la sauvegarde de la souveraineté suppose que l'État puisse déterminer avec suffisamment de précision les compétences transférées. Pourquoi?

La Cour constitutionnelle allemande estime que le programme d'intégration de l'Union européenne doit être suffisamment précis ${ }^{128} \mathrm{car}$ ce n'est pas le peuple lui-même qui est amené à consentir à ces transferts mais les pouvoirs publics. Or, ces derniers sont tenus par « la responsabilité pour l'intégration ${ }^{129}$. Ainsi, la validité des transferts est liée à la possibilité d'engager à chaque étape cette responsabilité ${ }^{130}$.

L'exigence de précision n'est pourtant pas totale. La Cour, lucide, concède que les organes des organisations internationales développent le programme de façon autonome dans une certaine limite. Ainsi, le champ d'action des institutions européennes ne peut pas être déterminé en totalité à l'avance. L'intégration suppose l'acceptation de la formation autonome d'une opinion des institutions de l'Union européenne. La Cour fixe également la marge " d'indéterminabilité » ${ }^{131}$. Elle refuse également que soit donné aux organes de I'Union un blanc seing. En effet, si les compétences sont transférées de façon indéterminée, les institutions européennes pourront agir au-delà des pouvoirs qui leur sont conférés. Sur ce point, on peut relever une incohérence. En effet, si le transfert est indéterminé, les institutions n'ont aucune limite à respecter. En revanche, lorsque la Cour explique qu'un transfert indéterminé ou indéterminable revient à conférer aux institutions européennes la compétence de la compétence, elle a raison. Sur ce point, l'atteinte à la souveraineté est consommée. Pour la Cour constitutionnelle allemande, le caractère précis et déterminable est fondé sur le respect du principe d'attribution et la responsabilité pour l'intégration ${ }^{132}$.

La condition tenant à la révocabilité se réalise dans la possibilité de retrait de l'Union, dont on a déjà expliqué à quel point elle était consubstantielle à la sauvegarde de la souveraineté 133 .

128 D. HANF, précit., p. 679.

129 BverfG 30 juin 2009, 2 BvE 2/08, Lisbon, § 236.

130 Ibidem, § 239.

131 BverfG 30 juin 2009, 2 BvE 2/08, Lisbon, § 237.

132 Ibidem, § 239.

133 Ibidem, $\S 231$ 
Les juges tchèques ont également posé la condition de précision, de caractère déterminable et contrôlable des transferts de compétences. Cette exigence est fondée sur des raisons similaires : l'État doit pouvoir prédire avec un degré suffisant de précision les compétences abandonnées parce que c'est la seule façon de déterminer s'il abandonne sa souveraineté. II doit pouvoir confronter ces compétences au material core protégé. Et pour sauvegarder sa souveraineté, il doit pouvoir en dernier lieu contrôler le respect par les institutions de l'Union des limites des transferts. La Cour est très souple, voire très favorable au traité dans l'application de ces conditions ${ }^{134}$. En effet, le Sénat tchèque reprochait aux articles 2 TFUE, $2 \S 6$ TFUE et $4 \S 2$ TFUE d'être libellés dans un sens qui ne permettait pas de déterminer avec suffisamment de précision et à l'avance le champ des compétences (exclusives et partagées) transférées. La Cour n'a pas souscrit à ce raisonnement, déclarant au contraire que les catégories en cause, qui ne sont pas nouvelles, étaient mieux organisées, ce qui constitue une amélioration ${ }^{135}$. Cette catégorisation renvoie à des dispositions particulières qui elles permettent de savoir avec précision quelle compétence est transférée. II n'y a donc pas de transfert illimité ${ }^{136}$. Un constat identique a été opéré à propos de l'article 216 TFUE dont la compatibilité avec l'article 10 a de la Constitution tchèque était douteuse. Cet article remplace la doctrine AETR de la Cour de justice ${ }^{137}$ à propos des compétences externes implicites. L'attitude de la Cour ne manque pas d'ambiguïté ${ }^{138}$. Tout en expliquant que cette disposition ne permet pas d'étendre les compétences de l'Union, elle concède que l'Union peut exercer sur ce fondement plus de compétences en externe qu'en interne ${ }^{139}$. Elle conclut à la constitutionnalité de cette disposition ${ }^{140}$ en précisant qu'elle est à la limite de ne pas remplir le critère de précision et de caractère déterminable ${ }^{141}$. Dans la décision Lisbonne II, les

134 P. BRIZA, précit., p. 153.

135 V. MICHEL, « Les compétences: les mots et les choses », Europe $\mathrm{n}^{\circ} 7$ juillet 2008, p. 43.

136 Décision du 26 novembre 2008, aff. PI. ÚS n 19/08, pt 133 et 134 ; P. BRIZA, précit., p. 152.

137 Dans son avis 1/94 du 15 novembre 1994, la Cour de justice développe sa doctrine AETR en précisant que la compétence externe exclusive de la Communauté ne découle pas ipso facto de son pouvoir d'édicter des règles sur le plan interne et que ce n'est que dans la mesure où des règles communes ont été établies sur le plan interne que la compétence de la Communauté devient exclusive.

138 P. BRIZA, précit., p. 158.

139 Décision du 26 novembre 2008, aff. PI. ÚS n 19/08, pt 183.

140 Ibidem, pt 185.

141 Ibidem, pt 186. 
requérants avaient mis en doute la compatibilité du droit de retrait avec l'article 10 a de la Constitution. D'après les requérants, si le transfert de pouvoirs doit être défini, la façon dont les mêmes pouvoirs seront réattribués doit également être définie ${ }^{142}$. Or, pour la Cour, le problème ne se pose pas en terme d'indétermination. Elle estime que les règles réalisent un équilibre entre les exigences de la souveraineté et celles liées au respect des engagements de la République tchèque envers les États membres ${ }^{143}$, et que l'argument des requérants repose sur une mauvaise compréhension du processus de retrait ${ }^{144}$.

À propos du contrôle juridictionnel, la Cour marque une grande confiance dans la juridiction de l'Union européenne. Ainsi, elle n'exercerait un contrôle du respect des limites des transferts que dans des cas exceptionnels ${ }^{145}$, un peu à la manière de la Cour constitutionnelle fédérale allemande dans son arrêt Solange II. Pour certains, la Cour minimise sa position à une possibilité théorique, en se fondant sur l'un des rares arrêts (Tobacco advertising ${ }^{146}$ ) où la Cour de justice a annulé une directive pour avoir excédé les limites des pouvoirs, et en exagérant le rôle des Parlements nationaux dans le processus de contrôle des compétences transférées ${ }^{147}$.

Les juges tchèques ont dégagé des critères de constitutionnalité des transferts similaires à ceux dégagés par les juges allemands, mais sont moins prolixes sur les fondements de ces critères et les liens qu'ils entretiennent avec la souveraineté. Cette similitude quant aux conditions se répercute logiquement sur les clauses contrôlées, qui sont pour la majeure partie les mêmes.

\section{b) L'application difficile des critères aux clauses sensibles}

Quatre dispositions du traité de Lisbonne affectent la « déterminabilité » des transferts car elles portent en germe de probables modifications du champ des compétences transférées. La clause de flexibilité de l'article 352 TFUE, la procédure de révision simplifiée, la clause générale de transition et les clauses passerelles ont donc été soumises à un examen exigeant.

\footnotetext{
142 Ibidem, pt 167.

143 Ibidem, pt 169.

144 Ibidem, pt 170.

145 Ibidem, pt 139.

146 CJCE, 5 octobre 2000, Allemagne contre Parlement et Conseil, aff. C-376/98, Rec. I8419.

147 P. BRIZA, précit., p. 152 et 153.
} 
- L'application des critères à la clause de flexibilité

La clause de flexibilité permet de combler les lacunes des compétences de l'Union. Une telle disposition porte en germe le ferment de l'évolution des compétences de l'Union dans une mesure aujourd'hui indéterminée et indéterminable. Elle ne répond donc pas aux conditions de précision et de " déterminabilité ».

Pourtant, le juge allemand a choisi de résoudre la contradiction en exigeant une ratification législative des actes adoptés sur cette base ${ }^{148}$.

Le juge tchèque a rendu un jugement plus favorable à la clause de flexibilité ${ }^{149}$. La Cour a procédé à un examen des conditions de mise en œuvre de cette clause ${ }^{150}$ et a constaté que les conditions étaient strictes et limitées pour empêcher une application disproportionnée de cette clause et a conclu à la compatibilité de l'article 352 TFUE avec l'ordre constitutionnel tchèque ${ }^{151}$. La Cour applique les critères et en déduit que le transfert n'est ni illimité ni insusceptible de contrôle. Mais ainsi que le souligne P. Briza, elle n'aborde pas explicitement la question de la spécificité qui est certainement le critère posant problème dans ce cas ${ }^{152}$.

La divergence d'interprétation d'une même clause par rapport à une même notion suscite l'interrogation. Cela tient sans doute à des politiques jurisprudentielles différentes. La Cour constitutionnelle allemande a un parti pris éminemment protecteur de la Loi fondamentale et a livré une décision particulièrement euro-sceptique alors que la Cour tchèque tente clairement de sauver le traité de Lisbonne. Des divergences similaires se retrouvent dans l'application des critères de constitutionnalité à la procédure de révision simplifiée.

- L'application des critères à la procédure de révision simplifiée

La procédure de révision simplifiée prévue à l'article 48 § 6 TUE a été examinée par les trois juges. La Cour tchèque l'a déclaré constitutionnelle sur le fondement de l'exigence d'une ratification et de la précision selon laquelle cette procédure ne peut pas attribuer de nouvelles compétences à I'Union ${ }^{153}$. La motivation de la Cour est pourtant ambivalente. Elle déclare d'une part que la révision simplifiée ne peut servir à accroître les compétences de l'Union ${ }^{154}$ et d'autre part, que

148 BverfG 30 juin 2009, 2 BvE 2/08, Lisbon, § 328, X. MAGNON, précit., p. 429.

149 Décision du 26 novembre 2008, aff. PI. ÚS n 19/08, pt 140.

150 Ibidem, pt 148, 149, 150.

151 Décision du 26 novembre 2008, aff. PI. ÚS n 19/08, pt 155.

152 P. BRIZA, précit., p. 156.

153 Décision du 26 novembre 2008, aff. PI. ÚS n 19/08, pt 164.

154 Ibidem, pt 160. 
les modifications intervenues sur le fondement de l'article $48 \S 6$ doivent être confrontées à l'article 10 a de la Constitution ${ }^{155}$. Or, s'il n'y a pas de transferts de pouvoirs, pourquoi exiger une confrontation avec l'article 10a de la Constitution? Enfin la Cour en appelle au pouvoir exécutif pour déterminer les modalités d'intervention du Parlement ${ }^{156}$.

Le Conseil constitutionnel déclare également cette procédure constitutionnelle pour des raisons similaires: la nécessité d'une ratification qui permettra le moment venu d'exercer un contrôle de constitutionnalité 157 .

La Cour constitutionnelle allemande en vient à la même conclusion mais précise que l'approbation parlementaire prendra la forme d'une loi ${ }^{158}$.

L'objet des futures révisions est par essence indéterminable, mais cela ne pose pas de problème car la ratification conserve la capacité de l'État à se déterminer. En ce sens, la souveraineté est sauvegardée.

- L'application des critères à la clause générale de transition

La clause générale de transition prévue à l'article 48 § 7 TUE a suscité des réserves chez les juges. Pour le juge français ${ }^{159}$, cette disposition appelait une révision de la Constitution car l'absence de procédure nationale de ratification rendait impossible un contrôle de constitutionnalité, alors que les accords internationaux doivent pouvoir faire l'objet d'un tel contrôle. L'intervention du Parlement national n'a pas été jugée suffisante, à raison. Son éventuelle opposition ne remédie pas à l'absence de contrôle de constitutionnalité. Ce contrôle est une nécessité pour assurer la compatibilité entre la Constitution et les normes internationales.

Pour le juge tchèque, cette clause générale de transition n'est pas inconstitutionnelle car il n'y a pas sur ce fondement un élargissement possible des compétences attribuées à l'Union ${ }^{160}$, car seules les

155 Ibidem, pt 167. Voir P. BRIZA, précit., p. 157 et 158 pour une explication et une solution de cette contradiction.

156 Décision du 26 novembre 2008, aff. PI. ÚS n 19/08, pt 165, 166.

157 CC, 560 DC, 20 décembre 2007, Traité modifiant le traité sur l'Union européenne et le traité instituant la Communauté européenne, pt 26. Le Conseil constitutionnel renvoie à sa précédente décision : CC, 505 DC, 19 novembre 2004, Traité établissant une Constitution pour l'Europe, pt 36.

158 BverfG 30 juin 2009, 2 BvE 2/08, Lisbon, § 312.

159 CC, 560 DC, 20 décembre 2007, Traité modifiant le traité sur l'Union européenne et le traité instituant la Communauté européenne, pt 27. Le Conseil constitutionnel renvoie à sa précédente décision: CC, 505 DC, 19 novembre 2004, Traité établissant une Constitution pour l'Europe, pt 35.

160 Décision du 26 novembre 2008, aff. Pl. ÚS nº 19/08, pt 161. 
modalités de vote sont concernées. La justiciabilité des actes permet de conclure que ce ne sont pas des transferts de pouvoirs selon l'article 10a. La Cour tchèque a une appréciation très souple de ces critères. S'il est vrai que les compétences de I'Union ne peuvent être étendues par ce biais, l'État perd tout de même un droit de veto lorsque l'unanimité cesse de s'appliquer et il perd un pouvoir d'influence au profit des institutions de l'Union lors du passage à la procédure législative ordinaire. Ainsi, en validant une clause dynamique, la Cour tchèque accepte aujourd'hui des atteintes futures à la souveraineté, sans que la République tchèque n'ait à se prononcer à nouveau. Cette validation va à l'encontre des critères de constitutionnalité dont le but est justement de cerner l'étendue et l'intensité des atteintes à la souveraineté pour pouvoir se décider en connaissance de cause. C'est donc une application assez curieuse des critères qu'elle a elle-même dégagé.

Pour le juge allemand, la clause générale de transition suppose une loi ${ }^{161}$ pour légitimer démocratiquement une telle décision ${ }^{162}$. L'indéterminabilité de certaines clauses pose un problème en terme de souveraineté car l'État ne peut appréhender les compétences abandonnées et en terme de démocratie. L'exigence d'approbation parlementaire y porte remède.

Ainsi, la clause générale de transition montre combien des critères similaires reçoivent une application différente suivant la politique jurisprudentielle constitutionnelle. La même considération vaut pour les clauses passerelles.

- L'application du principe aux clauses passerelles

Pour le juge constitutionnel français, les clauses passerelles sont inconstitutionnelles car l'absence de procédure de ratification empêche le contrôle de constitutionnalité ${ }^{163}$. II faut distinguer deux hypothèses. Si l'utilisation de la clause passerelle doit être adoptée à l'unanimité, la France peut refuser et la souveraineté est préservée par hypothèse. Dès lors, on comprend mal pourquoi le Conseil constitutionnel estime la disposition inconstitutionnelle. Peut être veut-il s'octroyer un droit de regard. Plus probablement, le juge constitutionnel est opposé au phénomène d'engrenage, à une construction qui fonctionne par anticipation, par des décisions qui ne feront l'objet d'aucun contrôle de

161 BverfG 30 juin 2009, 2 BvE 2/08, Lisbon, § 319.

162 X. MAGNON, précit., p. 429.

163 CC, 560 DC, 20 décembre 2007, Traité modifiant le traité sur l'Union européenne et le traité instituant la Communauté européenne, pt 23. F. LUCHAIRE, "La Constitution pour l'Europe devant le Conseil constitutionnel », RDP 1/2005, p. 56. 
constitutionnalité ${ }^{164}$. Or, la France doit pouvoir décider à chaque étape et non une fois pour toutes. Le Conseil constitutionnel souhaite un processus de ratification et un contrôle juridictionnel. En revanche, si la mise en œuvre de la clause passerelle requiert seulement la majorité, l'atteinte à la souveraineté est claire. Les clauses passerelles en question sont celles de l'article 31.3 TUE, 82.2 d) TFUE, 83.1 al 3 TFUE. Le Conseil constitutionnel maintient également l'exigence d'une révision constitutionnelle pour la clause passerelle en matière de droit de la famille (article 81.3 TFUE) qui permet à tout Parlement national de s'opposer à sa mise en œuvre ${ }^{165}$ car ce veto octroyé au Parlement n'équivaut pas à une procédure de ratification nationale.

Le juge allemand soumet l'utilisation des clauses passerelles à une décision des organes législatifs allemands ${ }^{166}$. Pour la clause passerelle de l'article 81.3 (2), il faudra une loi ${ }^{167}$. La responsabilité pour l'intégration et la nécessité de conservation une capacité à l'autodétermination expliquent cette exigence.

En ce qui concerne le juge tchèque, la décision ne porte que sur l'article 83.1 al 3 TFUE car les autres clauses passerelles n'ont pas été déférées. Cette disposition dynamique permet de définir de nouveaux domaines de criminalité transfrontalière. Le Sénat estime qu'une telle disposition porte atteinte à son rôle ${ }^{168}$. Or, la Cour constate qu'on ne peut appliquer cet article sans le consentement tchèque car il existe un mécanisme de protection des aspects fondamentaux du système de justice pénale des États membres. Ce mécanisme à lui seul suffit à la validation constitutionnelle de la disposition. Là encore, on constate à quel point la Cour est souple dans ses appréciations car un droit de veto est un pouvoir en négatif. Un droit de blocage ne suppose pas la même implication des organes législatifs qu'une ratification. Les clauses passerelles sont un dernier exemple de la variabilité de l'application de critères identiques.

II est néanmoins curieux de constater qu'un même traité confronté à une même notion suscite des réponses aussi diverses. Le point commun étant la ratification de ce traité dans tous les États. L'examen de la constitutionnalité du traité à l'aune de la démocratie révèle aussi quelques surprises.

164 L. FAVOREU et alii, Les grandes décisions du Conseil constitutionnel, 15e éd., Paris, Dalloz 2009, p. 765.

165 CC, 560 DC, 20 décembre 2007, Traité modifiant le traité sur l'Union européenne et le traité instituant la Communauté européenne, pt 25.

166 BverfG 30 juin 2009, 2 BvE 2/08, Lisbon, § 320.

167 BverfG 30 juin 2009, 2 BvE 2/08, Lisbon, § 319, X. MAGNON, précit., p. 430.

168 Décision du 26 novembre 2008, aff. PI. ÚS nº 19/08, pt 168. 


\section{II - L'APPRECIATION DE L'ATTEINTE A LA DEMOCRATIE}

Dans les trois décisions, la notion de démocratie apparaît. Dans la décision française, la souveraineté nationale (article $3 \mathrm{DDHC}$ ), la souveraineté populaire (article 3 de la Constitution) et le principe de démocratie représentative ${ }^{169}$ font partie des normes de référence du contrôle. Les requêtes allemande et tchèque mettaient en doute la conformité du traité de Lisbonne au caractère démocratique de l'État ${ }^{170}$.

Le Président de la République tchèque estime notamment qu'en l'absence de peuple européen, il n'existe pas de source légitime du pouvoir en Europe. Dès lors, il assimile l'Union à une sorte de cheval de Troie qui permettra de faire pénétrer dans l'ordre juridique des États membres des normes que les Parlements nationaux auraient refusées. L'ampleur des transferts, combinée au manque de légitimité démocratique de I'Union, porte atteinte à la souveraineté nationale ${ }^{171}$. Pour le Sénat, c'est l'article $83 \S 1$ TFUE qui pose un problème au regard de la démocratie parlementaire car les Parlements nationaux ne sont pas impliqués dans le processus de définition des activités criminelles, l'implication du Parlement européen n'étant pas suffisante à cet égard 172.

Les requérants allemands invoquaient la violation de l'article 20.1 de la Loi fondamentale relatif au caractère démocratique de l'État et de l'article 20.2 de la Loi fondamentale sur la souveraineté populaire : "Le principe démocratique, pour autant qu'il est intangible en vertu de l'article $79 \S 3$, LF, serait en effet méconnu à deux égards : les compétences législatives du Bundestag allemand seraient vidées de leur substance, d'une part, et l'Union européenne ne disposerait pas de la nécessaire légitimation, d'autre part " ${ }^{173}$. Ils invoquaient également la violation de l'article 38.1 de la Loi fondamentale qui impliquerait un droit à la démocratie. Ce droit serait violé car l'ampleur des transferts ne permettrait plus au citoyen allemand de légitimer l'exercice de l'autorité

169 CC, 560 DC, 20 décembre 2007, Traité modifiant le traité sur l'Union européenne et le traité instituant la Communauté européenne, pt 4.

170 Décision du 26 novembre 2008, aff. PI. ÚS n 19/08, pt 2, 8, 10 pour la souveraineté populaire, 168.

171 Ibidem, pt 61.

172 Décision du 26 novembre 2008, aff. PI. ÚS n 19/08, pt 168.

173 BverfG 30 juin 2009, 2 BvE 2/08, Lisbon, § 100,101 traduction de K. M. BAUER, précit., p. 801. 
publique, d'autant que cette possibilité ne se retrouve pas non plus au niveau européen ${ }^{174}$.

À la lecture des décisions, on constate que la démocratie est un élément de l'identité constitutionnelle des États membres. C'est à ce titre qu'il fait partie des normes de référence du contrôle de constitutionnalité, c'est à ce titre qu'il est invoqué par les requérants allemand et tchèque, c'est à ce titre qu'il est protégé par les juges constitutionnels. Cette convergence sur la nature de ce principe n'empêche pas une profonde divergence quant à son application.

\section{A - La démocratie, élément de l'identité constitutionnelle des États membres}

En France, on peut rattacher la démocratie à la République ${ }^{175}$. La République est le parachèvement historique de la démocratie pluraliste et représentative, avec toutes les caractéristiques liées à la République : une, indivisible, laïque. La démocratie s'accomplit dans la République. II y a une continuité historique de la République puisqu'à partir de la IIIe République, la démocratie parlementaire est un élément permanent de l'État. C'est parce que démocratie parlementaire et République sont liées dans l'histoire constitutionnelle française que ce principe constitue un élément de l'identité constitutionnelle française. L'article 3 de la Constitution est la forme juridiquement exprimée de la démocratie.

En Allemagne, les principes formant l'identité constitutionnelle sont inscrits dans la clause d'éternité, l'article $79 \S 3$ de la Loi fondamentale. La Cour constitutionnelle rattache le principe de la démocratie et le droit de vote à cette clause ${ }^{176}$.

En République tchèque, le caractère démocratique de l'État est consacré à l'article 1er de la Constitution. Pour la Cour, cet article comprend les caractéristiques essentielles de l'État. Elle fait de ce principe la norme de référence centrale ${ }^{177}$.

Les trois décisions font place à l'analyse de l'impact du traité de Lisbonne sur le caractère démocratique de l'État. Elles diffèrent sur le

174 BverfG 30 juin 2009, 2 BvE 2/08, Lisbon, § 100, 104, 105, 106, 107, 127, 128, 132, $133,135,168$.

175 La République est entendue à la fois comme une forme de gouvernement et l'État, comme structure juridique et institutionnelle.

176 BverfG 30 juin 2009, 2 BvE 2/08, Lisbon, § 206, 208, 216. Voir D. DouKAS, précit., p. 872, C. SCHÖNBERGER, précit., p. 1208 (l'auteur critique le rattachement du principe démocratique à la clause d'éternité), F. SCHORKOPF, précit.,, p. 1223, D. HANF, précit., p. 680 , X. MAGNON, précit., p. 425.

177 Décision du 26 novembre 2008, aff. PI. ÚS n 19/08, pt 93 et 97. 
contenu de l'analyse mais convergent sur le résultat car tous les juges constitutionnels conviennent que le traité de Lisbonne ne porte pas atteinte au respect de ce principe.

\section{B - La compatibilité du traité avec les exigences démocratiques}

Pour la Cour constitutionnelle fédérale allemande, le respect du principe démocratique suppose que les transferts de compétences ne vident pas de sa substance le pouvoir des organes législatifs allemands d'une part et d'autre part que l'exercice de l'autorité publique au niveau de l'Union soit légitimée démocratiquement.

Ainsi, dans sa première dimension, le respect du principe démocratique est consubstantiellement lié à la souveraineté. "La souveraineté est conçue comme ayant deux dimensions complémentaires englobant l'indépendance externe de l'État et un pouvoir de décision interne. Le jugement Lisbonne lie les deux perspectives et les intègre dans l'autorité ultime du peuple et son droit à l'autodétermination démocratique " ${ }^{178}$. La Cour explique que l'élection du Bundestag n'a d'intérêt que si ce dernier exerce une influence suffisante sur la politique ${ }^{179}$. Donc, le principe de démocratie suppose que soient fixées des limites liées au contenu, au transfert des pouvoirs souverains ${ }^{180}$.

Dans sa seconde dimension, le principe démocratique impose ses exigences à la construction européenne. Ainsi, la Cour analyse l'étendue des pouvoirs transférés et le degré d'émancipation des procédures décisionnelles européennes.

II y a un lien de proportionnalité ${ }^{181}$ entre les pouvoirs transférés à l'Union et le niveau de légitimation démocratique de l'Union. Ainsi, plus le transfert est important, plus la légitimité démocratique de l'Union doit être effective ${ }^{182}$. Mais la Cour nuance son appréciation. Estimant que I'Union exerce une autorité publique dérivée, elle n'a pas à se conformer aux exigences démocratiques étatiques ${ }^{183}$. Le respect de l'article $23 \S 1$ de la Loi fondamentale n'exige pas une compatibilité structurelle ${ }^{184}$.

178 D. THYM, précit., p. 1798.

179 BverfG 30 juin 2009, 2 BvE 2/08, Lisbon, § 246.

180 BverfG 30 juin 2009, 2 BvE 2/08, Lisbon, § 247.

181 X. MAGNON, précit., p. 426, A. VON UNGERN-STERNBERG, précit., p. 174.

182 BverfG 30 juin 2009, 2 BvE 2/08, Lisbon, § 263.

183 BverfG 30 juin 2009, 2 BvE 2/08, Lisbon, § 271.

184 D. HANF, précit., p. 682. 
Son analyse l'amène à déclarer le traité de Lisbonne compatible avec le principe démocratique ${ }^{185}$ pour plusieurs raisons. D'une part, le peuple décide toujours des questions essentielles. Cette solution était inévitable en raison de l'adéquation entre la liste des compétences insusceptibles de transferts et celles des compétences restant à l'État allemand. D'autre part, le degré de légitimation de l'Union européenne est, en l'état actuel de sa construction (c'est-à-dire dans un système d'attribution limitée des compétences) suffisant. La conclusion est presque surprenante tant la Cour est critique vis-à-vis du rôle du Parlement européen ${ }^{186}$ et tant elle sous-estime l'impact des mécanismes de démocratie directe et participative ${ }^{187}$ existants dans l'Union ${ }^{188}$. Cette conclusion est également l'issue d'un raisonnement complexe ${ }^{189}$ au cours duquel elle analyse l'Union à l'aune de critères inadéquats. En effet, le cadre d'analyse de la Cour est purement étatique ${ }^{190}$ alors que I'Union est une construction sui generis ${ }^{191}$. Par ailleurs, le modèle de démocratie égalitaire et majoritaire tel qu'il est présenté par la Cour relève plus de la théorie que de la réalité constitutionnelle des démocraties en Europe ${ }^{192}$.

Cette conclusion est ambivalente. Elle est positive car elle permet la ratification du traité de Lisbonne aujourd'hui. Mais, elle a une portée négative car elle hypothèque la construction européenne en l'enfermant dans un cadre étatique ${ }^{193}$.

Les juges tchèques concluent également au respect du principe démocratique mais à l'issue d'un raisonnement différent. Le Sénat

185 BverfG 30 juin 2009, 2 BvE 2/08, Lisbon, § 274.

186 P. KIIVER, "Reflexions on the Lisbon judgment - how the judges at Karlsruhe trust neither the European Parliament nor their national Parliament », Maastricht journal of European and comparative law 2009, vol. 16, $\mathrm{n}^{\circ} 3$, p. 265 et s, D. DoukAS, précit., p. 885 ; M. NiedOBITECK, "The Lisbon case of 30 June 2009 - A comment from the European law perspective ", The German Law Journal, vol. $10, n^{\circ} 8$, p. 1272 ; C. SCHÖNBERGER, précit., p. 1213, D. THYM, précit., p. 1812, D. HANF, précit., p. 706. BverfG 30 juin 2009, 2 BvE 2/08, Lisbon, § 271 et 274.

187 Article 11 TUE. Pour une appréciation de son importance par la Cour constitutionnelle fédérale allemande : BverfG 30 juin 2009, 2 BvE 2/08, Lisbon, § 295.

188 D. DOUKAS, précit., p. 885.

189 Pour une critique du raisonnement tenu par la Cour, lire A. VON UNGERN-STERNBERG, précit., p. 180.

190 Voir sur ce point l'analyse des institutions européennes, BverfG 30 juin 2009, 2 BvE 2/08, Lisbon, § 280-297, X. MAGNON, précit., p. 417.

191 C. SCHÖNBERGER, précit., p. 1210 ; D. DOUKAS, précit., p. 884.

192 C. SCHÖNBERGER, précit., p. 1210 ets ; D. THYM, précit., p. 1814-1815 ; D. DOUKAS, précit., p. 885, C. TOMUSCHAT, précit., p. 1260 ; D. HANF, précit., p. 707.

193 D. HANF, précit., p. 685 ; X. MAGNON, précit., p. 433 ets. 
craignait surtout une perte du pouvoir législatif ${ }^{194}$. Ainsi, l'essentiel de l'argumentation tient dans l'affirmation que le traité de Lisbonne implique suffisamment les parlements nationaux ${ }^{195}$. Ainsi, en ce qui concerne l'article $83 \S 1$ TFUE qui est l'objet de la requête du Sénat, le juge tchèque rappelle que le parlement national peut remplir son rôle d'examen préliminaire d'après les dispositions pertinentes du protocole sur l'application des principes de subsidiarité et proportionnalité 196 pour conclure au respect du principe démocratique. La Cour fait cependant appel au pouvoir exécutif en précisant la responsabilité particulière du gouvernement dans la préparation et l'adoption des règles juridiques qui permettront l'application complète de ces pouvoirs ${ }^{197}$. La divergence méthodologique avec le juge allemand est flagrante. Ce dernier ne se contente pas de faire un appel solennel aux pouvoirs publics, mais impose une intervention des organes législatifs allemands par le biais des lois d'accompagnement.

Ainsi, les juges constitutionnels ont déclaré le traité de Lisbonne conforme au principe démocratique. Cette issue est importante car elle conditionnait la ratification du traité. Mais ces déclarations de compatibilité n'ont pas la même valeur. Le juge tchèque n'a pas examiné la question en profondeur. Seule une disposition a été confrontée au principe démocratique, alors que le juge allemand a analysé l'intégralité du traité de Lisbonne. De plus, les exigences sont très variables. Ainsi, le juge tchèque se fonde essentiellement sur l'implication des parlements nationaux pour conclure au respect du principe démocratique, alors que les juges allemands ont une grille d'analyse qui comprend non seulement cette participation, mais également celle du Parlement européen et des citoyens ${ }^{198}$.

La confrontation du principe démocratique à la structure institutionnelle et décisionnelle de l'Union souffre d'un problème méthodologique. Le cadre d'appréciation de la démocratie est à l'heure actuelle l'État. On voit bien la difficulté qu'il y a à appliquer des concepts stato-centrés à une entité non étatique. La question du peuple européen est patente à cet égard. La citoyenneté de l'Union est insuffisante pour créer un peuple européen. II n'existe pas de peuple européen, donc il n'existe pas de sujet de légitimation démocratique. Or, à partir du

194 Décision du 26 novembre 2008, aff. $\mathrm{n}^{\circ} \mathrm{PI}$. Ús 19/08, pt 168. Les juges font ici référence à l'article 12 TUE qui énonce la manière dont les parlements nationaux contribuent activement au fonctionnement de l'Union.

195 Décision du 26 novembre 2008, aff. n Pl. ÚS 19/08, pt 173.

196 Ibidem, pt 175.

197 Ibidem, pt 174.

198 Cf. art. 11 TUE. 
moment où on pourra identifier un peuple européen, on aura déjà l'un des éléments constitutifs d'un futur État européen. Le schéma de pensée reste celui de l'État. C'est parce que la Cour allemande applique rigoureusement le principe démocratique à l'Union qu'elle en vient à cette conclusion sans issue. En effet, l'Union souffre d'un déficit démocratique, mais il est acceptable car l'Union n'est pas un État et ce déficit est compensé par l'exercice de la démocratie au niveau national. Mais combler ce déficit en augmentant les pouvoirs du Parlement européen en tant que source autonome de légitimation démocratique violerait la garantie constitutionnelle de la démocratie dans la mesure où ce serait un abandon de souveraineté ${ }^{199}$. L'Union ne peut devenir pleinement démocratique qu'en se transformant en un État (et en faisant les modifications appropriées).

L'application trop rigoureuse de principes stato-centrés réduit l'avenir de l'Union à la seule hypothèse de l'État. A l'opposé, une application trop souple de ces principes confine à une absence de garanties au profit du citoyen.

Les décisions constitutionnelles rendues à propos du traité de Lisbonne confirment la difficulté à appréhender la construction européenne par le prisme étatique. L'État est une figure en cours de mutation. II faut assouplir les concepts qui le sous-tendent pour trouver un équilibre entre la nécessaire poursuite de la construction européenne et la perpétuation des États européens. 